\title{
The DNA-polymorphism rs849142 is associated with skin toxicity induced by targeted anti-EGFR therapy using cetuximab
}

\author{
Matthias F. Froelich ${ }^{1}$, Sebastian Stintzing ${ }^{2,3,4}$, Jörg Kumbrink ${ }^{1,3,4}$, Thomas G.P. \\ Grünewald $^{1,4,6}$, Ulrich Mansmann ${ }^{4,5}$, Volker Heinemann ${ }^{2,3,4}$, Thomas Kirchner ${ }^{1,3,4}$ \\ and Andreas Jung $1,3,4$ \\ ${ }^{1}$ Institute of Pathology, Medical Faculty, LMU Munich, Munich, Germany \\ ${ }^{2}$ Department of Medicine III, University Hospital LMU Munich, Munich, Germany \\ ${ }^{3}$ Comprehensive Cancer Center, University Hospital LMU Munich, Munich, Germany \\ ${ }^{4}$ German Cancer Research Centre (DKFZ); German Cancer consortium (DKTK), Heidelberg, Germany \\ ${ }^{5}$ Institute for Medical Informatics, Biometry, and Epidemiology, University of Munich, Munich, Germany \\ ${ }^{6}$ Max-Eder Research Group for Pediatric Sarcoma Biology, Institute of Pathology, Medical Faculty, LMU Munich, Munich, \\ Germany
}

Correspondence to: Andreas Jung, email: andreas.jung@/mu.de

Keywords: colorectal cancer; SNPs; skin toxicity

Received: December 29, $2017 \quad$ Accepted: June 12, $2018 \quad$ Published: July 13, 2018

Copyright: Froelich et al. This is an open-access article distributed under the terms of the Creative Commons Attribution License 3.0 (CC BY 3.0), which permits unrestricted use, distribution, and reproduction in any medium, provided the original author and source are credited.

\section{ABSTRACT}

Skin toxicity (ST) is a frequent adverse effect (AE) in anti-epidermal growth factor receptor (EGFR)-targeted treatment of metastatic colorectal cancer (MCRC) resulting in decreased quality of life and problems in clinical management. We wanted to identify biomarkers predicting ST in this setting and focused on 70 DNA polymorphisms associated with acne, the (immunoglobulin fragment crystallizable region) Fcy-receptor pathway, and systemic lupus erythematosus (SLE) applying next-generation-sequencing (NGS). For the analysis patients with mCRC treated with cetuximab were selected from the FIRE-3 study. A training group consisting of the phenotypes low (1) - and high-grade (3) ST $(n=16)$ and a validation group $(n=55)$ representing also the intermediate grade (2) were genotyped and investigated in a genotype-phenotype association analysis. The single nucleotide polymorphism (SNP) rs849142 significantly associated with ST in both the training- $(p<0.01)$ and validation-group $(p=0.04)$. rs849142 is located in an intron of the juxtaposed with another zinc finger protein 1 (JAZF1) gene. Haplotype analysis demonstrated significant linkage disequilibrium of rs849142 with JAZF1. Thus, rs849142 might be a predictive biomarker for ST in anti-EGFR treated $\mathrm{MCRC}$ patients. Its value in the clinical management of AE has to be validated in larger cohorts.

\section{INTRODUCTION}

Skin toxicities (ST) like papulopustular rash (acneiform eruption), erythema, skin fissures, hair and nail changes, paronychia, and xerosis are frequent adverse effects (AE) when targeting the EGFR in solid cancers like metastatic colorectal cancer (mCRC), lung cancer, head and neck cancers and others [1]. ST occurs mostly at the face, scalp and trunk within the first three weeks or during the second cycle of treatment with variable severity in $82 \%$ of $\mathrm{mCRC}$-patients receiving targeted anti-EGFRtreatment [2-5]. 38-70\% of the patients also demonstrated bacterial but seldom viral infections at sites of ST $[2,6]$ all of which negatively affected quality of live [2, 7-9]. ST is treated pre-emptively and symptomatically applying special creams as well as oral and topical treatment [7, 10]. It is unknown if these compounds interfere with antiEGFR targeted therapy. The severity of ST is classified by a simple three tier grading system -Common Toxicity Criteria for Adverse Events (CTCAE)-: grade 1 (mild; 
$\sim 40 \%$ of the patients), grade 2 (moderate, $\sim 40 \%$ ), and grade 3 (severe, $\sim 20 \%$ ) (National Cancer Institute; CTCAE. http://evs.nci.nih.gov/) [3]. Interestingly, ST is an on-treatment marker for the efficacy of the anti-EGFR targeted therapy $[2,5,8,9,11]$. Despite its acneiform appearance, it differs both etiologically and pathologically from acne as for example comedones are missing [7]. Reasons for ST might be alterations in the cytokine/ chemokine profile, disturbed keratinocyte differentiation, barrier defects due to xerosis or infection [2]. Predictive biomarkers for ST were investigated and include a polymorphism in intron 1 of the EGFR-gene influencing expression levels [9, 12], expression of inflammatory lymphokines [13], and serum levels of EGFR-ligands [14-16]. Unfortunately, the predictive value of these biomarkers is either low or was not validated. Thus, none of these biomarkers found its way into clinical practice. Therefore, we searched for biomarkers which are predictive for ST in $\mathrm{mCRC}$ and identified the rs849142 SNP as a potentially predictive biomarker for ST in antiEGFR treated mCRC patient cohort of the FIRE-3 study.

\section{RESULTS}

\section{Skin toxicity is significantly associated with survival in a FIRE-3 study collective}

As only a limited number of specific biomarkers predicting ST is available which has not been approved for clinical use [16] our aim was to identify and validate potentially clinically useful predictive biomarkers for ST. Therefore, we chose a sub-collection of mCRC patients from the FOLFIRI plus cetuximab arm of the FIRE-3 study $[17,18]$. A training/ validation approach was chosen including 16 randomly selected $\mathrm{mCRC}$ patients with ST grade 1 or 3 for the training group (mean age 64.2 years) and 55 patients in the validation group (mean age 62.4 years) with ST grade 1, 2, and 3 (Table 1). To see if this subpopulation of the FIRE-3 study still represented the complete group of patients we tested for the known correlation of the ST-grade induced by anti-EGFR therapy with the survival of the patients [9] employing KaplanMeier statistics. In the chosen subgroup ST grade and survival statistically correlated with both progression free survival (PFS; $p<0.001$ ) and overall survival (OS; $p<0.001)$ comparably to the whole patient collective (Figure 1). Similar results were also obtained when the training- and validation sets were tested independently (PFS, $p$-training set $=0.016, p$-validation set $=0.004$; OS, $p$-training set $=0.059, p$-validation set $<0.004)$ (Supplementary Figure 1).

Thus, representative subgroups of patients of the clinical FIRE-3 study were available which were therefore enrolled as the data-basis for subsequent steps in the identification of predictive biomarkers for anti-EGFR therapy induced ST.

\section{SNPs known to be associated with acne, $F c R \gamma$, and SLE were selected for the investigation of ST in $\mathrm{mCRC}$ patients treated with cetuximab}

Because ST is most likely a tumor-independent AE mediated by anti-EGFR antibody treatment, we hypothesized that single nucleotide polymorphisms (SNP) which are known to be related to other ST like acne [1921], systemic lupus erythematodes (SLE) [22], or FcR $\gamma$ signaling $[23,24]$ might also be indicative for anti-EGFR treatment related ST. Appropriate markers were selected from the literature [6, 19-24] and public databases (https:// www.snpedia.com) (Figure 2). All together 14 SNP associated with acne, 20 SNPs with FcR $\gamma$, and 36 with SLE were identified as potential markers for ST (Figure 2). Six of these SNPs were found in both the SLE- as well as the FcR $\gamma$-subgroup. Thus, together 64 SNPs were employed in an NGS-approach for the investigation of ST in mCRC patients treated with anti-EGFR antibodies. After multiple steps of NGS quality control testing (Supplementary Figure 2) a set of 61 SNPs and 71 patients remained for the linkage analysis of SNPs associated with ST.

\section{SNP rs849142 is significantly associated with ST related to cetuximab}

To investigate a possible association between genotypes (SNPs) and severity of ST the genetic linkage analysis routine of the PLINK software [25] was applied for both the training- and validation set (Figure 3). It turned out that rs849142 (T allele) was significantly associated with ST in both the training- $(p=0.004$, Sens $=100.00 \%$, Spec $=83.33 \%, \mathrm{PPV}=80.00 \%, \mathrm{NPV}=100.00 \%$, Figure $3 \mathrm{~A})$ and validation group $(p=0.0436$, Sens $=86.36 \%$, Spec $=44.44 \%, \mathrm{PPV}=79.17 \%, \mathrm{NPV}=57.14 \%$, Figure $3 \mathrm{~B})$ as well as in the whole patient collective $(p=0.004$, Supplementary Figure 3). In contrast rs849142 ( $\mathrm{T}$ allele) only correlated significantly with PFS in the training group ( $p=0.05$ ) (Supplementary Figure 4) but neither with OS in the training- nor PFS and OS in the validation group. rs849142 did not deviate significantly from HardyWeinberg equilibrium ( $p=0.44$, Supplementary Table 2). Also another SNP, rs463426, significantly correlated with ST in the validation- $(p=0.036)$ and whole group $(p<0.001)$, but showed only a trend for statistical significance in the training group $(p=0.066)$. Therefore, rs463426 was excluded from further analyses.

Global allele frequencies of $\operatorname{rs} 849142(\mathrm{~T} ; \mathrm{C})$ are given as T: $0.765, \mathrm{C}: 0.235, \mathrm{~T}|\mathrm{~T}: 0.615, \mathrm{C}| \mathrm{C}: 0.086, \mathrm{C} \mid \mathrm{T}$ : 0.299 (1000 Genomes Project) showing strong variations in different ethnicities (for details refer to Figure 4A). The European population displays almost balanced allele frequencies (T: 0.492, C: 0.508) compared to the East Asian population (T: $0.985, \mathrm{C}: 0.015)$. Our results (Table 2) are expectedly in line with this data-set for the European subpopulation (T: $0.419, \mathrm{C}: 0.581$ ) which is 
Table 1: Composition of the evaluated training and validation patient groups

\begin{tabular}{lccccccc}
\hline \multirow{2}{*}{ Group } & Age & \multicolumn{2}{c}{ Sex } & \multicolumn{3}{c}{ Skin toxicity } \\
\cline { 2 - 7 } & mean & male & female & unknown & $\mathbf{1}$ & $\mathbf{2}$ & $\mathbf{3}$ \\
\hline Training $(\boldsymbol{n}=\mathbf{1 6})$ & 64.2 & $6(37.50 \%)$ & $7(43.75 \%)$ & $3(18.75 \%)$ & $11(68.75 \%)$ & $0(0.00 \%)$ & $5(31.25 \%)$ \\
Validation $(\boldsymbol{n}=\mathbf{5 5})$ & 62.4 & $43(78.18 \%)$ & $12(21.82 \%)$ & $0(0.00 \%)$ & $19(34.55 \%)$ & $16(29.09 \%)$ & $20(36.36 \%)$ \\
\hline
\end{tabular}

another indicator for the support for the correctness of our measurements (Figure 4A). It turned out that the T-allele especially in the homozygous form $(\mathrm{T} \mid \mathrm{T})$ indicates high grade ST (Table 2).

\section{rs849142 is located in the JAZF1 gene}

In a next step, we wanted to know if the genomic localization of rs849142 might help to elucidate a possible molecular mechanism. Therefore, database searches and in silico analyses were performed. rs849142 is located on chromosome 7 within an intronic region of the Juxtaposed with Another Zinc Finger protein 1 (JAZF1) gene (Figure 4B). JAZF1 is a transcriptional regulator that directly represses transcription or acts as a corepressor [26]. JAZF1 was not shown to be directly associated with ST but one of its interaction partners, Transforming growth factor $\beta$-activated kinase 1 (TAK1) is an integral component of signaling pathways involved in modulating the immune response [27]. The intronic position of rs849142 does not allow to conclude on a potential influence on transcriptional regulation of the $J A Z F 1$ gene. Unfortunately, no skin samples from our patient collective or other ST studies were available to measure the $J A Z F 1 \mathrm{mRNA}$ expression profile. It is known that $J A Z F 1$ is ubiquitously expressed with highest levels in the adrenal gland and whole blood and lowest expression in the ST/ immune responseassociated organs skin, spleen, and thyroid (expression

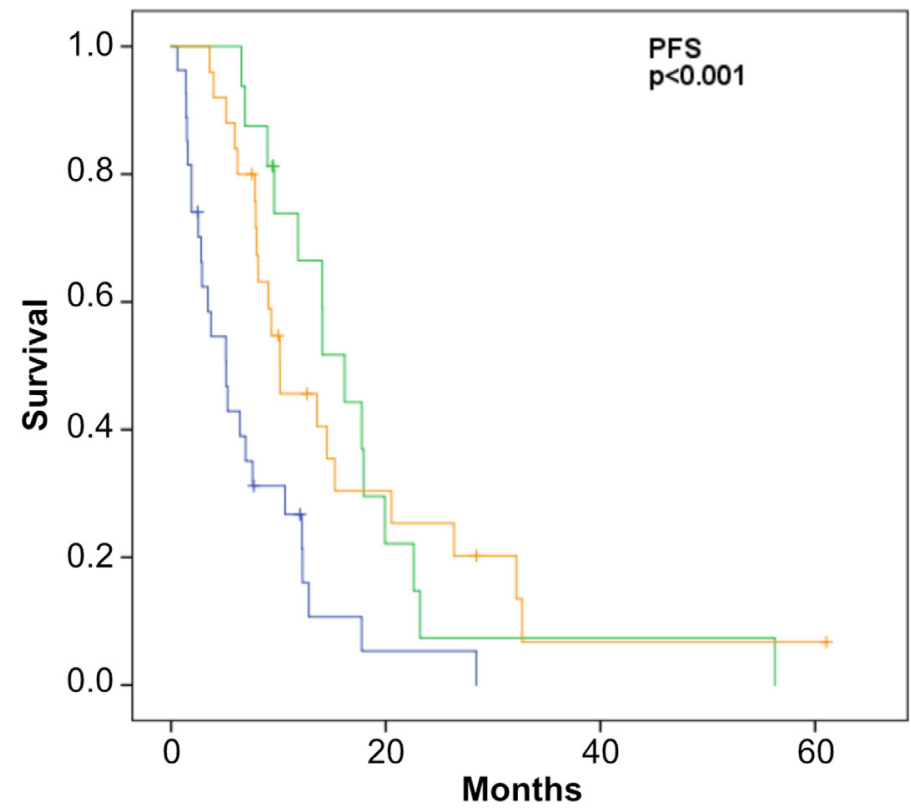

data from: gtexportal.org (2)) (Figure 4C). Additionally, the expression of $J A Z F 1$ correlates inversely with the levels of the cetuximab target EGFR in the skin and whole blood (Figure 4C). Taken together, these results suggest that $J A Z F 1$ itself may not be directly involved in the development of cetuximab-mediated ST.

To explore a potential bystander effect indicated by linkage of rs849142 with other genes a linkage analysis of SNPs neighboring rs849142 was performed (Figure 5). rs849142 was only found in a coupling group with the SNPs rs864745 and rs849140 which are both located in the $J A Z F 1$ gene. Therefore, rs849142 is also in linkage disequilibrium (LD) with several other SNPs within $J A Z F 1$ and the adjacent non-protein coding JAZF1-AS1 (antisense RNA 1) gene (Figure 4B). But also JAZF1-AS1 has not been associated with ST, thus the connection of the SNP rs849142 and ST remains unknown like mutations in the FOXL2 gene in granulosa cell tumors of the ovary [28]. Definitely, more research has to be invested.

\section{DISCUSSION}

In our study collective, the SNP rs849142 (T allele) was identified and validated as a potential biomarker for skin-related adverse effects in mCRC patients under targeted anti-EGFR treatment using cetuximab (FIRE-3 study). Thus, genotyping of the rs849142 might help to improve decision-making on prophylactic ST treatment in

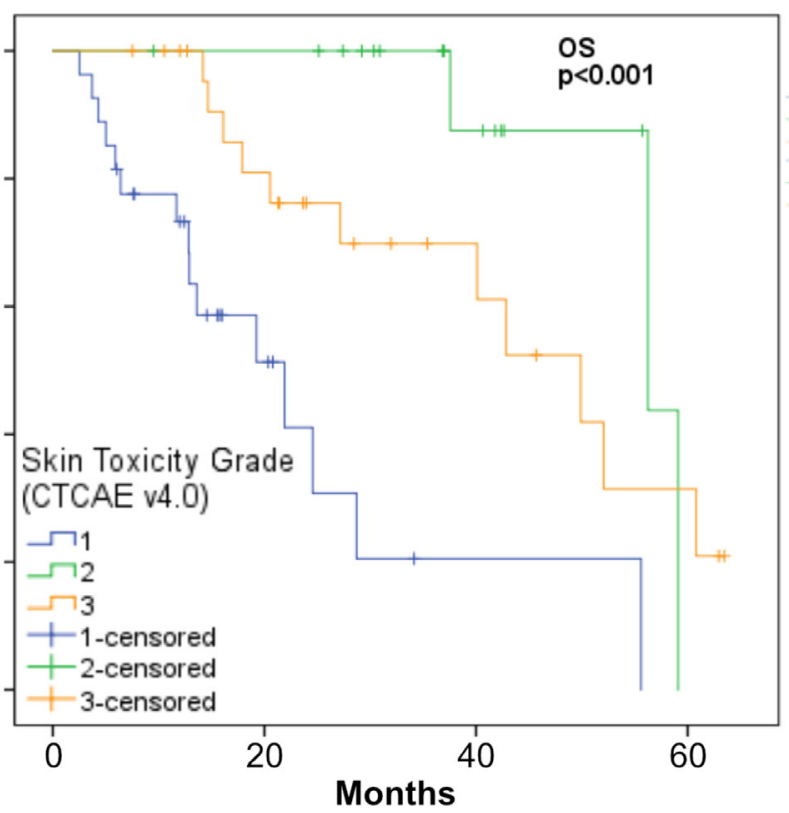

Figure 1: PFS and OS Kaplan-Meier plots for cetuximab associated ST grades. 
Table 2: Distribution of rs841942 in the patient collective

\begin{tabular}{|c|c|c|c|c|}
\hline Training & ST Grade 1 & ST Grade 2 & ST Grade 3 & Sum \\
\hline No variant call & $5(83.33 \%)$ & $0(0 \%)$ & $1(16.67 \%)$ & 6 \\
\hline $\mathrm{C} / \mathrm{C}$ & $5(100 \%)$ & $0(0 \%)$ & $0(0 \%)$ & 5 \\
\hline $\mathrm{C} / \mathrm{T}$ & $1(20 \%)$ & $0(0 \%)$ & $4(80 \%)$ & 5 \\
\hline $\mathrm{T} / \mathrm{T}$ & $0(0 \%)$ & $0(0 \%)$ & $0(0 \%)$ & 0 \\
\hline Sum & 11 & 0 & 5 & 16 \\
\hline Validation & ST Grade 1 & ST Grade 2 & ST Grade 3 & Sum \\
\hline No variant call & $1(33.33 \%)$ & $0(0 \%)$ & $2(66.67 \%)$ & 3 \\
\hline $\mathrm{C} / \mathrm{C}$ & $8(57.14 \%)$ & $3(21.43 \%)$ & $3(21.43 \%)$ & 14 \\
\hline $\mathrm{C} / \mathrm{T}$ & $8(27.59 \%)$ & $11(37.93 \%)$ & $10(34.48 \%)$ & 29 \\
\hline $\mathrm{T} / \mathrm{T}$ & $2(22.22 \%)$ & $2(22.22 \%)$ & $5(55.56 \%)$ & 9 \\
\hline Sum & 19 & 16 & 20 & 55 \\
\hline
\end{tabular}

the situation of targeted anti-EGFR treatment of left-sided and wildtypic (WT) KRAS- and NRAS gene mCRCs. In such a scenario, the high-risk group of patients with a $\mathrm{T} \mid \mathrm{T}$ constellation of rs849142 might be considered for prophylactic ST treatment. As rs849142 is a genetic marker its testing has several advantages: First, higher sensitivity and specificity than measurements of variable day-to-day parameters like mRNA or serum components
[13] and serum-level markers (AREG, EREG, HGF). Second, SNP detection can be measured easily from liquid biopsies (blood, saliva) and is less invasive than taking tissue biopsies. Third, SNP-typing can be performed together with the testing for mutations in the KRAS- und NRAS-genes as the predictive test for using anti-EGFR targeting antibodies in the therapy of mCRC. Due to the different global distribution of rs849142 alleles in distinct

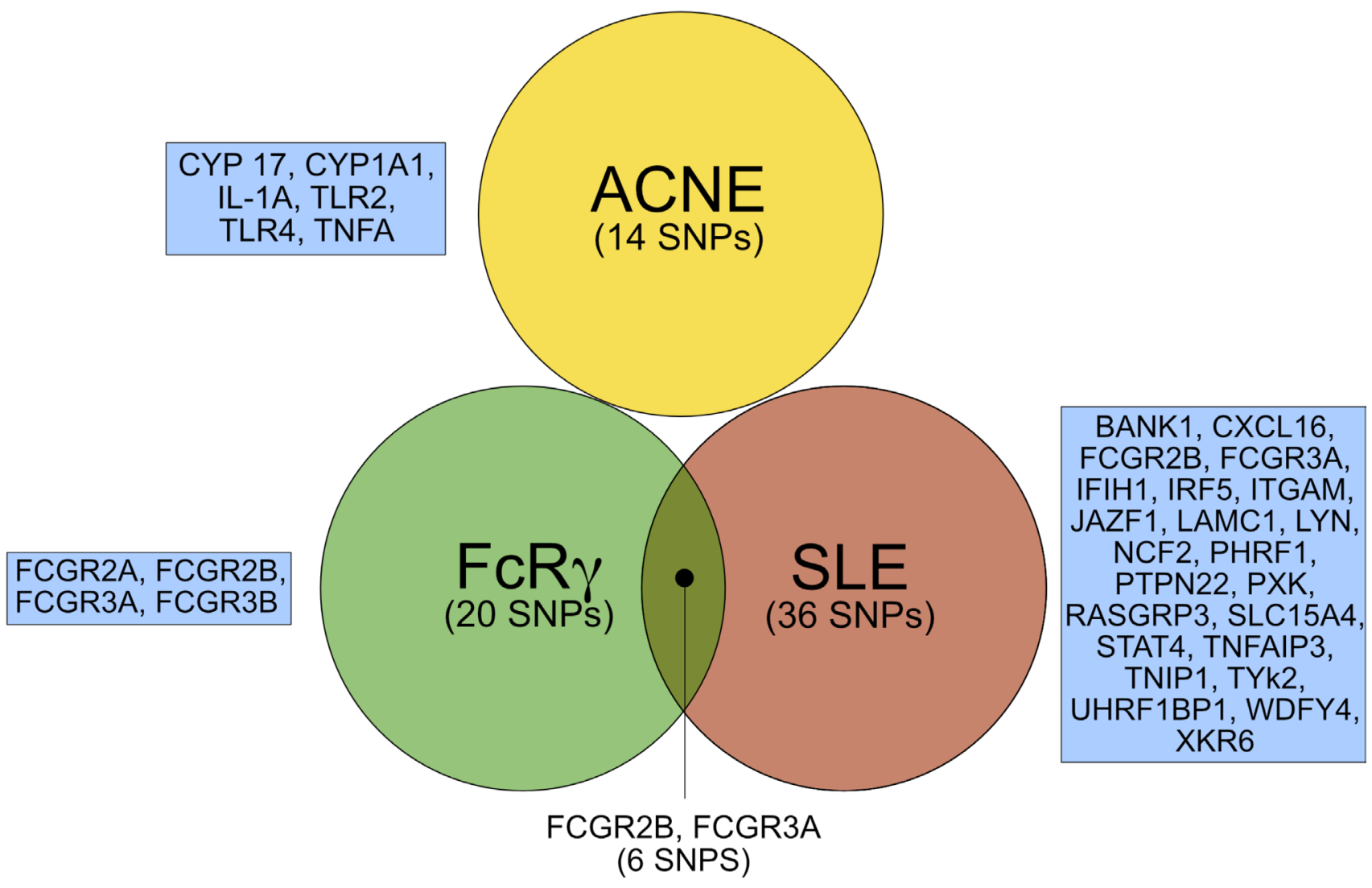

Figure 2: SNPs and respective genes selected for targeted sequencing (complete results in Supplementary Table 1). FcR $\gamma$, Fc receptor $\gamma$; SLE, systemic lupus erythematodes. SNP; single nucleotide polymorphism. 
A

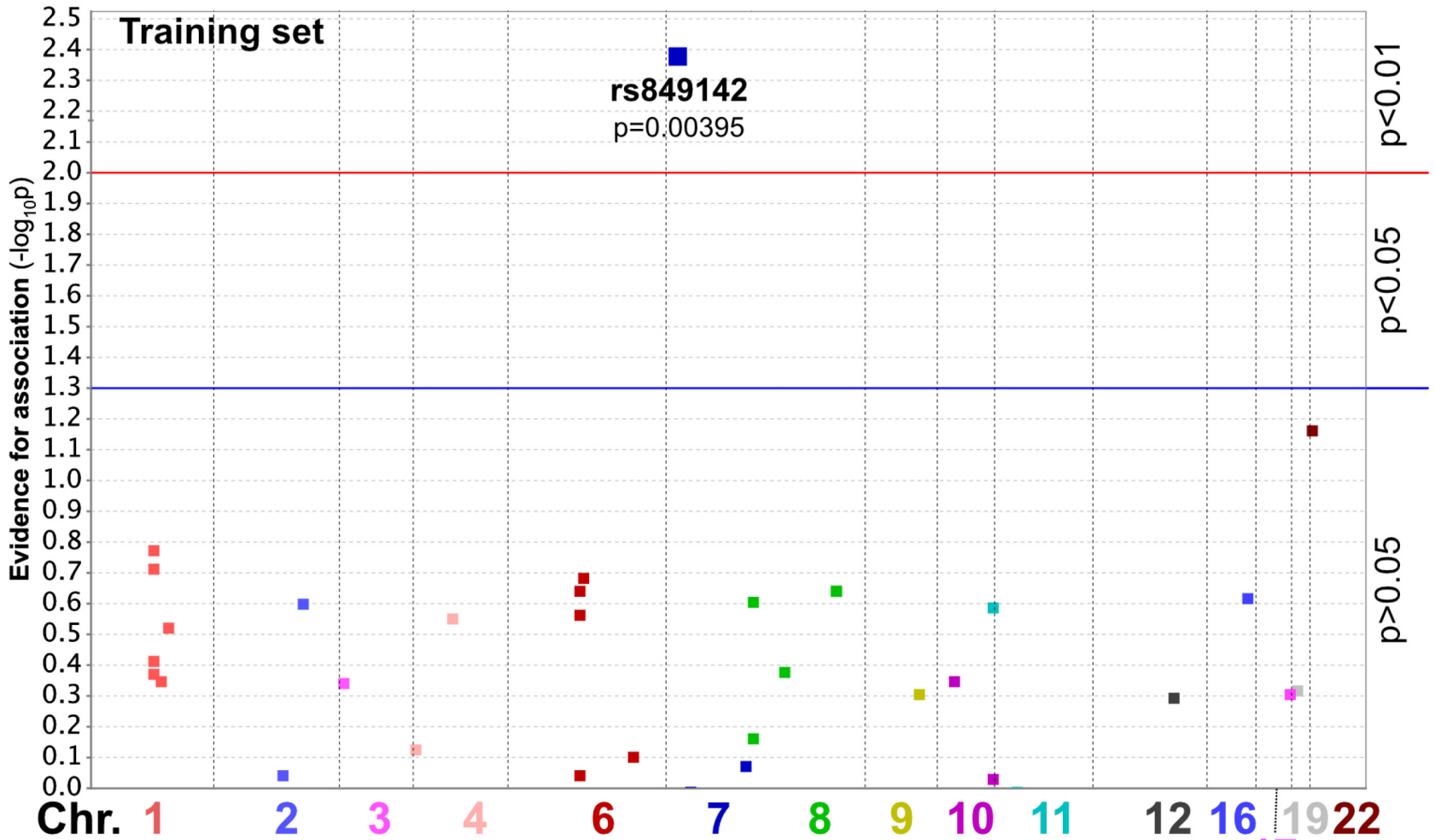

B

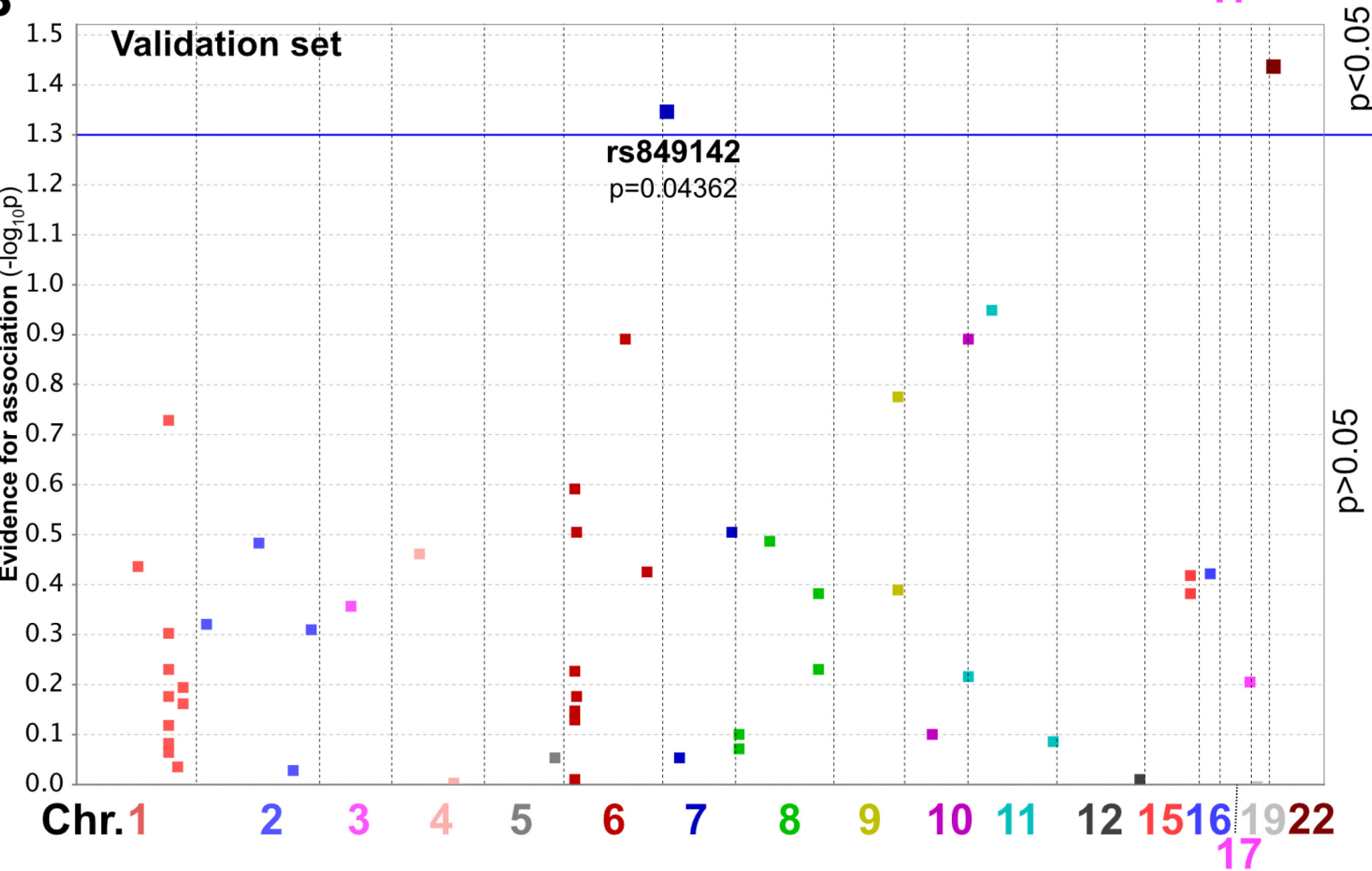

Figure 3: Manhattan plot for ST of patients from the Training group (A) or Validation group (B). SNP position on the chromosome is indicated by horizontal position. Y-axis value describes magnitude of evidence for association calculated as $-\log _{10}(p$ value). Chr., chromosome. 
ethnicities SNP-testing might be especially valuable for Europeans (T: 49.2\%, C: 50.8\%) and Americans (T: 65\%, C: 35\%) but less for Africans (T: $84.9 \%$, C:15.1\%) and especially East Asians (T: $98.5 \%, \mathrm{C}: 1.5 \%$ ). Due to the small size of our collections, the missing correlation with survival as well as underling molecular mechanism, these results are preliminary. Further research especially validation in larger cohorts is essential.

For our aim to find biomarkers predicting ST in antiEGFR treatment of mCRC patients, we chose a training/ validation approach in a patient collective of the FIRE-3 study $[17,18]$. Our kind of approach includes a high bias as the statistic variance is high due to the low amounts of events. For this reason seldom events might be missed. The alternative is a linkage analysis which requires numbers of events in the range of several hundreds to thousands. It is questionable if such high numbers of patients with documented ST grade are accessible even globally. Another major drawback of small collections might be a drift in the representativity compared to the whole collection. Therefore, we confirmed by applying an additional ST unrelated parameter, namely the response to anti-EGFR

A
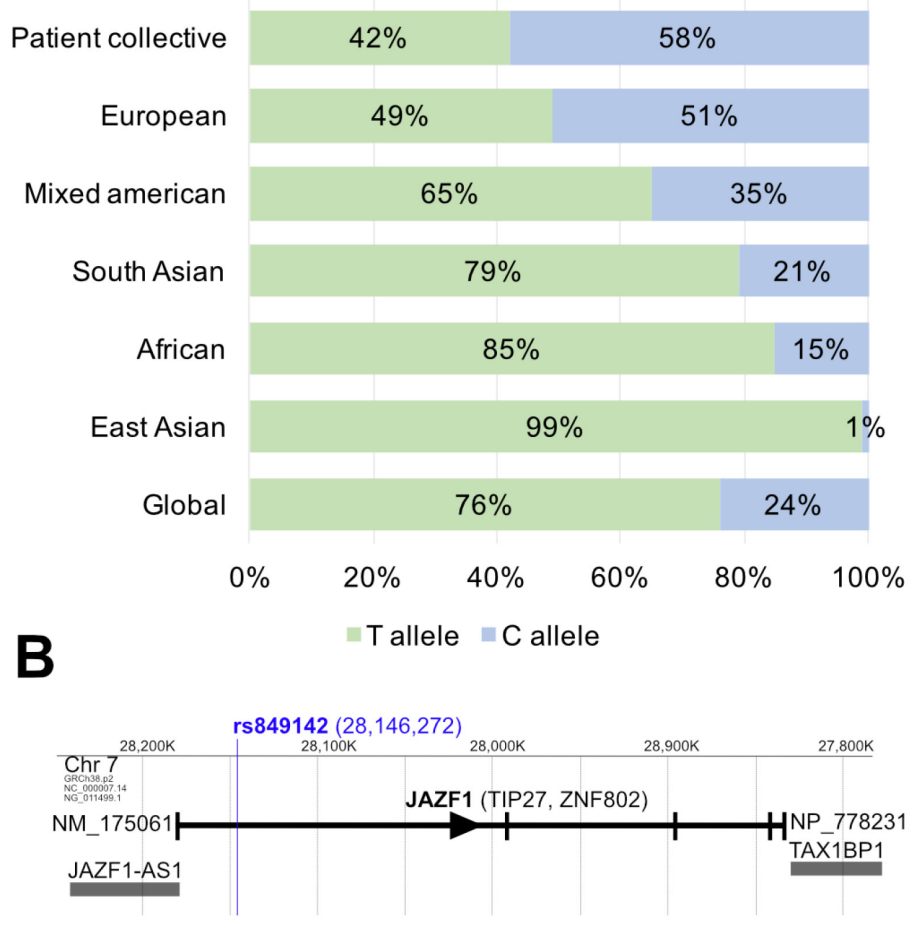

directed therapy [9], that our sub-selection still reflected the whole FIRE-3 patient cohort. Another bias in training/ validation approaches is the need for an input of preselected parameters; in our case the collection of SNPs under investigation. Therefore, three non-oncological diseases were chosen, because ST in mCRC patients is most likely a tumor independent effect. 1: Acne is a common disease linked to deregulation and changes in the skin environment [29]. 2: SLE is an autoimmune disease that commonly results in skin-related manifestations [30]. 3: Imunoglobulin fragment crystallizable receptors $(\mathrm{FcR})$ are responsible for unspecific binding of antibody-classes via the Fc-region of the antibody which might result in the joining of tumorwith other (immune) cells eventually resulting in antibodydependent cell-mediated cytotoxicity (ADCC) or other kinds of interference. SNPs are known to influence the affinity of FcRs for immunoglobulins [31]. As cetuximab is an IgG1 antibody the class of FcR $\gamma$ was of interest for our study. This approach resulted in a total of 61 SNPs which are known to modulate acne, SLE, and FcR $\gamma$ binding. Of these only rs849142 - found in the SLE group — was significantly associated with ST in both the training and validation group.

C
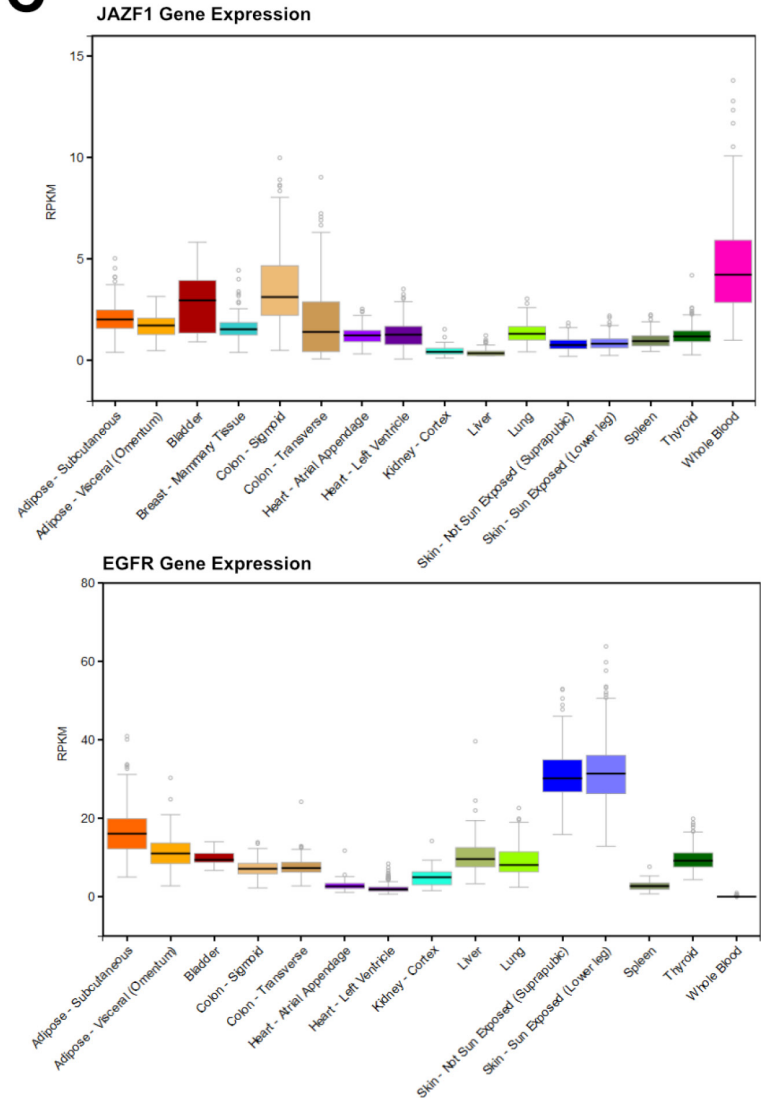

Figure 4: (A) Global- and sub-population allele frequencies of rs849142 (T>C) in comparison to the FIRE-3 patient collective as annotated at 1000genomes.org. (B) JAZF1 genomic region on Chromosome 7. JAZF1 and adjacent genes are shown. Position of rs849142 is indicated by the blue line. Reading direction of $J A Z F 1$ indicated by an arrowhead. Vertical bars indicate exons. JAZF1, Juxtaposed with another zinc finger protein 1. JAZF1-AS1, JAZF1 antisense RNA 1. (C) mRNA expression of JAZF1 (upper panel) and EGFR (lower panel) in various and ST related tissues as obtained from http://www.gtexportal.org. Expression is presented in RPKM (reads per kilobase of transcript per million mapped reads). 
As ST correlates with survival of mCRC patients [9], it was reasonable to check if this was also true for rs849142. Unexpectedly, a significant association of rs849142 ( $\mathrm{T}$ allele) was only observed for PFS in the training group. No correlation was present with OS in the training set nor PFS and OS in the validation group. Thus, ST and response or survival seem to share several but differ in other characteristics. Underlying molecular mechanisms are unknown and thus arguing would be speculative. Moreover, as rs849142 is located in the intron of $J A Z F 1$ aside from functional relevant sites like splice donor/acceptor it is idle to discuss a possible role or function. For sure both open questions need further research.

Taken together, our study revealed rs849142 as a potentially predictive biomarker of ST in cetuximab treated mCRC patients. Patients may largely benefit from a prediction of ST and potentially the severity of the disease by genotyping rs849142 as physicians could manage ST in the relevant population of patients thereby treating only that subset of patients where ST will occur as an AE of the anti-EGFR targeted treatment. Moreover, reading out the genotype of the rs 849142 alleles can easily be incorporated into the detection pipeline for the analysis of other relevant molecular biomarkers for mCRC, namely mutations in the BRAF-, KRAS- and NRAS-genes as well as microsatellite instability [32]. Finally, it is important to note that our finding has to be validated in a larger cohort or at best in the setting of a prospective clinical study.

\section{MATERIALS AND METHODS}

\section{Patients}

The FIRE-3 study was a randomized phase 3 study of stage IV mCRC patients (age 18-75 years) treated with the combinatorial-chemotherapy (CTX) folinic acid, leucovorin, 5-Fluoruracil (5-FU), and Irinotecan (FOLFIRI) with either cetuximab or bevacizumab. The inclusion criteria for FIRE-3 study have been described in detail before [17]. Further inclusion criteria for this particular analysis were: Appropriate follow-up data, cetuximab treatment group presence of remaining DNA samples. ST grades were determined using the common

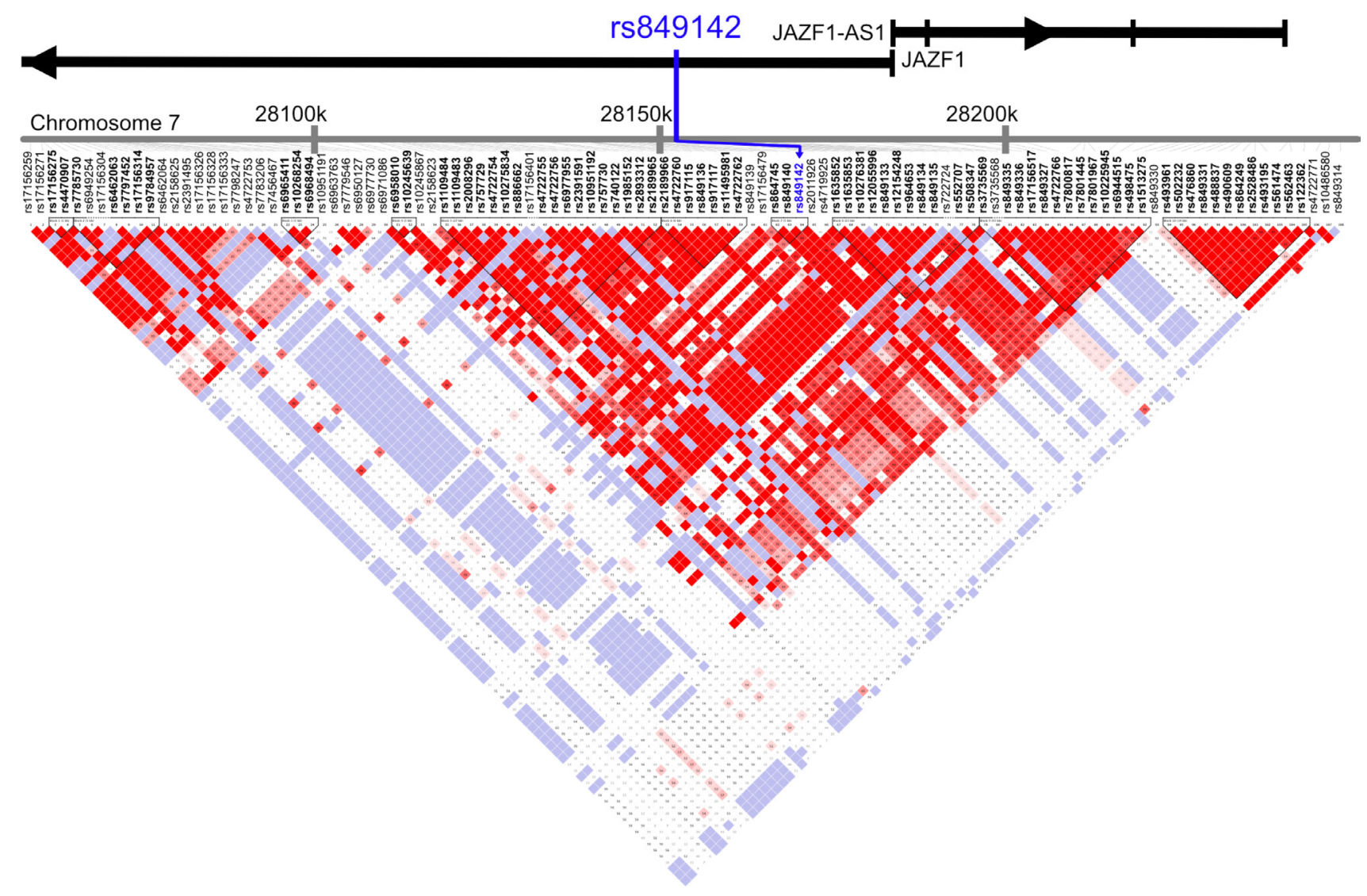

Figure 5: Linkage Disequilibrium (LD) Plot of Chromosome $728150 \mathrm{kB} \pm 100 \mathrm{kB}$ using Version 2 release 24 CEU (CEPH, Utah residents with ancestry from northern and western Europe). SNPs are presented in their genomic order. rs 849142 is indicated by blue arrow. The reading direction (arrowhead) and exons (vertical bars) of the JAZF1 and the partially overlapping $J A Z F 1-A S 1$ genes are indicated on top. The degree of positive correlation between two SNPs is visualized by color-coded squares at the intersection of orthogonal lines originating from each variant: White: low LD, low confidence; Shades of pink/red: low LD, high confidence; blue: high LD, low confidence; bright red: high LD, high confidence. 
toxicity criteria of adverse events (CTCAE) version 4.0. For a training-validation approach, two subgroups of the cetuximab treatment arm were analyzed: 1) training group $(n=16)$ consisting of patients with grade one and three ST; 2) validation group $(n=55)$ including patients with ST grade one, two, and three. For this approach, from all eligible patients showing ST grade 1 or 3 , sixteen were assigned randomly to the training group. All other eligible patients were included into the validation group before the analysis.

\section{DNA extraction and genotyping}

Genomic DNA was isolated from peripheral blood mononuclear cells using QIAGEN blood kits following the vendor's recommendations and used as the template for a targeted next-generation-sequencing (NGS) approach together with custom sequencing panels. Therefore, three Ion Ampliseq ${ }^{\mathrm{TM}}$ Custom DNA-Hotspot panels were designed utilizing Ion Torrent Ampliseq ${ }^{\circledR}$ Custom Panel Designer (Supplementary Table 1). They were used together with Ampliseq ${ }^{\mathrm{TM}}$ Library kits for preparing libraries which were analyzed on an NGS IonTorrent PGM $^{\text {TM }}$ (Personal Genome Machine, Thermo Fisher Scientific) platform. The experimental procedures were done according to the manufacturer's manual. Briefly, DNA concentration and quality was measured using the Qubit ${ }^{\mathrm{TM}} 3$ Fluorimeter (Thermo Fisher Scientific). The concentration of amplifiable DNA was quantified using an RNase P-gene specific detection system (Roche Diagnostics). Samples with a ratio of $>2$ were extracted again. Amplicon libraries were created using the Ion Ampliseq ${ }^{\mathrm{TM}}$ Library Kit 2.0 (Thermo Fisher Scientific) with 21 PCR cycles. For comparable coverage of the samples, DNA concentration was controlled for by the Ion Torrent qPCR quantification following the vendor's instructions. Groups of 16 libraries were transferred into the IonChef ${ }^{\mathrm{TM}}$ pipetting station for clonal amplification by emulsion PCR and Ion-316 ${ }^{\mathrm{TM}}$ Chip loading. Chips were loaded onto the IonTorrent PGM ${ }^{\mathrm{TM}}$ (Personal Genome Machine, Thermo Fisher Scientific).

\section{Statistical analysis}

Analysis of data obtained by NGS was done applying the Ion Reporter ${ }^{\mathrm{TM}}$ v.4.6 software: data quality was controlled for by application of filter criteria recommended by the vendor. Reads were mapped to the reference genome hg19 and variants were called using the standard settings. SNP annotation was based on dbSNP138. Resulting variant calling files (VCF) were reformatted to match specifications of the PLINK software version 1.07 [25]. Of note, SNPs showing more than two alleles were excluded from the analysis, because they cannot be evaluated in a typical quantitative genetic association analysis. Overview tables on quality based on patients and variants were calculated using the summary statistics function of the
PLINK software. The fraction of successfully genotyped variants was visualized in Haploview version 4.2 (Supplementary Figures 3 and 4) [33]. The exact HardyWeinberg equilibrium (HWE) test module of PLINK was used for calculating deviations from HWE applying a significance level of $p<0.001$. Genotype-phenotypeassociation was estimated for each group using the Wald statistic in PLINK measuring ST as a quantitative marker. Results were visualized as Manhattan plots (Haploview software). Sensitivity [Sens], specificity [Spec], positive predictive value [PPV] and negative predictive value [NPV] were calculated comparing the genotype corresponding to ST grade 1 and to all other genotypes and ST grades. Linkage disequilibrium analysis of a region spanning $100 \mathrm{~kb}$ upstream and downstream of rs849142 was run with the Haploview software and haplotype blocks were calculated [34]. Pooled over strata $p$-values of survival analyses were calculated with the SPSS 23.0 software using log-rank- and Kaplan-Meier models.

\section{Abbreviations}

ADCC: antibody-dependent cell-mediated cytotoxicity; AE: adverse effect; CTCAE: Common Toxicity Criteria for Adverse Events; CTX: combinatorialchemotherapy; EGFR: epidermal growth factor receptor; FcR: Imunoglobulin fragment crystallizable receptors; FOLFIRI: chemotherapy scheme folinic acid: leucovorin: 5-Fluoruracil (5-FU): and Irinotecan; HWE: HardyWeinberg equilibrium; JAZF1: juxtaposed with another zinc finger protein 1; mCRC: metastatic colorectal cancer; NGS: Next-Generation-Sequencing; NPV: negative predictive value; OS: overall survival; PFS: progression free survival; PPV: positive predictive value; Sens: sensitivity; SLE: systemic lupus erythematosus; SNP: single nucleotide polymorphism; Spec: specificity; ST: skin toxicity.

\section{Author contributions}

Conception and design: MFF, AJ; Development of methodology: MFF, AJ; Acquisition of data: MFF, SS, AJ; Analysis and interpretation of data: MFF, JK, AJ, TGPG; Writing, review, and/or revision of the manuscript: MFF, JK, AJ; Administrative, technical, or material support: SS, VH; Study supervision: SS, JK, VH, TK, AJ, UM.

\section{ACKNOWLEDGMENTS}

We thank Gertrud Lenz, Sabine Sagebiel-Kohler, and Sebastian Heucke for their outstanding technical support.

\section{CONFLICTS OF INTEREST}

M.F.F. declared no conflict of interest. S.S. has received honoraria for talks, advisory boards and travel 
expenses by RocheAG, Merck-Serono KgaA, Amgen, Bayer, Lilly, Sanofi and Sirtex. J.K. declared no conflict of interest. T.G.P.G. declared no conflict of interest. U.M. has received travel support by Baxter. V.H. has received honoraria from Merck-Serono KGaA, Roche AG, Amgen, Sanofi, SIRTEX and BAXALTA and has received travel support from Merck-Serono KGaA, Roche AG, Amgen, SIRTEX and Baxalta and has served on advisory boards for Merck-Serono KGaA, Roche AG, Amgen, Sanofi, Lilly, SIRTEX, Böhringer Ingelheim, Baxalta, Taiho and Merrimack. T.K. received honoria for taking part in advisory boards and giving talks for Amgen, AstraZenca, BMS, Merck-Serono KGaA, Pfizer, Roche KGaA. A.J. received honoraria for taking part in advisory boards and giving talks for Amgen, AstraZenca, BMS, Merck-Serono KGaA, Pfizer, Qiagen, Roche AG.

\section{FUNDING}

This work was supported by the DKTK (German Cancer Consortium), Heidelberg, Germany (www.dkfzdktk.de). The FIRE-3 study was sponsored by the University Hospital Großhadern, Ludwig-Maximilians-University (LMU) Munich and received financial support from Merck Serono KGaA, Darmstadt, Germany, Pfizer Pharma GmbH, Karlsruhe, Germany and DKTK, Heidelberg, Germany.

TGPG is supported by grants from the German Cancer Aid (DKH-111886 and DKH-70112257).

\section{REFERENCES}

1. Kozuki T. Skin problems and EGFR-tyrosine kinase inhibitor. Jpn J Clin Oncol. 2016; 46:291-98. https://doi. org/10.1093/jjco/hyv207.

2. Holcmann M, Sibilia M. Mechanisms underlying skin disorders induced by EGFR inhibitors. Mol Cell Oncol. 2015; 2:e1004969. https://doi.org/10.1080/23723556.2015.1004969.

3. Bergman H, Walton T, Del Bel R, Seki JT, Rafii A, Xu W, Koren G, Shear N, Krzyzanowska MK, Howell D, Liu G. Managing skin toxicities related to panitumumab. J Am Acad Dermatol. 2014; 71:754-59. https://doi.org/10.1016/j. jaad.2014.06.011.

4. Thaler J, Karthaus M, Mineur L, Greil R, Letocha H, Hofheinz R, Fernebro E, Gamelin E, Baños A, Köhne CH. Skin toxicity and quality of life in patients with metastatic colorectal cancer during first-line panitumumab plus FOLFIRI treatment in a single-arm phase II study. BMC Cancer. 2012; 12:438. https:// doi.org/10.1186/1471-2407-12-438.

5. Jaka A, Gutiérrez-Rivera A, López-Pestaña A, del Alcázar E, Zubizarreta J, Vildosola S, Arregui MA, Sarasqueta C, Lobo C, Tuneu A. Predictors of Tumor Response to Cetuximab and Panitumumab in 116 Patients and a Review of Approaches to Managing Skin Toxicity. Actas Dermosifiliogr. 2015; 106:483-92. https://doi.org/10.1016/j.adengl.2015.05.014.
6. Eilers RE Jr, Gandhi M, Patel JD, Mulcahy MF, Agulnik M, Hensing T, Lacouture ME. Dermatologic infections in cancer patients treated with epidermal growth factor receptor inhibitor therapy. J Natl Cancer Inst. 2010; 102:4753. https://doi.org/10.1093/jnci/djp439.

7. Lacouture ME, Mitchell EP, Piperdi B, Pillai MV, Shearer H, Iannotti $\mathrm{N}, \mathrm{Xu} \mathrm{F}$, Yassine $\mathrm{M}$. Skin toxicity evaluation protocol with panitumumab (STEPP), a phase II, open-label, randomized trial evaluating the impact of a pre-Emptive Skin treatment regimen on skin toxicities and quality of life in patients with metastatic colorectal cancer. J Clin Oncol. 2010; 28:1351-57. https://doi.org/10.1200/JCO.2008.21.7828.

8. Stintzing S, Fischer von Weikersthal L, Vehling-Kaiser U, Stauch M, Hass HG, Dietzfelbinger H, Oruzio D, Klein S, Zellmann K, Decker T, Schulze M, Abenhardt W, Puchtler G, et al. Correlation of capecitabine-induced skin toxicity with treatment efficacy in patients with metastatic colorectal cancer: results from the German AIO KRK-0104 trial. Br J Cancer. 2011; 105:206-11. https://doi.org/10.1038/bjc.2011.227.

9. Stintzing S, Kapaun C, Laubender RP, Jung A, Neumann J, Modest DP, Giessen C, Moosmann N, Wollenberg A, Kirchner T, Heinemann V. Prognostic value of cetuximabrelated skin toxicity in metastatic colorectal cancer patients and its correlation with parameters of the epidermal growth factor receptor signal transduction pathway: results from a randomized trial of the GERMAN AIO CRC Study Group. Int J Cancer. 2013; 132:236-45. https://doi.org/10.1002/ijc.27654.

10. Boone SL, Rademaker A, Liu D, Pfeiffer C, Mauro DJ, Lacouture ME. Impact and management of skin toxicity associated with anti-epidermal growth factor receptor therapy: survey results. Oncology. 2007; 72:152-59. https:// doi.org/10.1159/000112795.

11. Hu J, Zhang Z, Zheng R, Cheng L, Yang M, Li L, Liu B, Qian $\mathrm{X}$. On-treatment markers as predictors to guide anti-EGFR $\mathrm{MoAb}$ treatment in metastatic colorectal cancer: a systematic review with meta-analysis. Cancer Chemother Pharmacol. 2017; 79:275-85. https://doi.org/10.1007/s00280-016-3196-2.

12. Graziano F, Ruzzo A, Loupakis F, Canestrari E, Santini D, Catalano V, Bisonni R, Torresi U, Floriani I, Schiavon G, Andreoni F, Maltese P, Rulli E, et al. Pharmacogenetic profiling for cetuximab plus irinotecan therapy in patients with refractory advanced colorectal cancer. J Clin Oncol. 2008; 26:1427-34. https://doi.org/10.1200/JCO.2007.12.4602.

13. Vallböhmer D, Zhang W, Gordon M, Yang DY, Yun J, Press OA, Rhodes KE, Sherrod AE, Iqbal S, Danenberg KD, Groshen S, Lenz HJ. Molecular determinants of cetuximab efficacy. J Clin Oncol. 2005; 23:3536-44. https://doi. org/10.1200/JCO.2005.09.100.

14. Takahashi N, Yamada Y, Furuta K, Honma Y, Iwasa S, Takashima A, Kato K, Hamaguchi T, Shimada Y. Serum levels of hepatocyte growth factor and epiregulin are associated with the prognosis on anti-EGFR antibody treatment in KRAS wild-type metastatic colorectal cancer. 
Br J Cancer. 2014; 110:2716-27. https://doi.org/10.1038/ bjc.2014.230.

15. Takahashi N, Yamada Y, Furuta K, Nagashima K, Kubo A, Sasaki Y, Shoji H, Honma Y, Iwasa S, Okita N, Takashima A, Kato K, Hamaguchi T, Shimada Y. Association between serum ligands and the skin toxicity of anti-epidermal growth factor receptor antibody in metastatic colorectal cancer. Cancer Sci. 2015; 106:604-10. https://doi.org/10.1111/cas.12642.

16. Kubo A, Hashimoto H, Takahashi N, Yamada Y. Biomarkers of skin toxicity induced by anti-epidermal growth factor receptor antibody treatment in colorectal cancer. World J Gastroenterol. 2016; 22:887-94. https://doi.org/10.3748/ wjg.v22.i2.887.

17. Heinemann V, von Weikersthal LF, Decker T, Kiani A, Vehling-Kaiser U, Al-Batran SE, Heintges T, Lerchenmüller C, Kahl C, Seipelt G, Kullmann F, Stauch M, Scheithauer $\mathrm{W}$, et al. FOLFIRI plus cetuximab versus FOLFIRI plus bevacizumab as first-line treatment for patients with metastatic colorectal cancer (FIRE-3): a randomised, open-label, phase 3 trial. Lancet Oncol. 2014; 15:1065-75. https://doi.org/10.1016/S1470-2045(14)70330-4.

18. Stintzing S, Modest DP, Rossius L, Lerch MM, von Weikersthal LF, Decker T, Kiani A, Vehling-Kaiser U, Al-Batran SE, Heintges T, Lerchenmüller C, Kahl C, Seipelt $\mathrm{G}$, et al, and FIRE-3 investigators. FOLFIRI plus cetuximab versus FOLFIRI plus bevacizumab for metastatic colorectal cancer (FIRE-3): a post-hoc analysis of tumour dynamics in the final RAS wild-type subgroup of this randomised openlabel phase 3 trial. Lancet Oncol. 2016; 17:1426-34. https:// doi.org/10.1016/S1470-2045(16)30269-8.

19. Bataille V, Snieder H, MacGregor AJ, Sasieni P, Spector TD. The influence of genetics and environmental factors in the pathogenesis of acne: a twin study of acne in women. J Invest Dermatol. 2002; 119:1317-22. https://doi. org/10.1046/j.1523-1747.2002.19621.x.

20. Bhate K, Williams HC. Epidemiology of acne vulgaris. Br J Dermatol. 2013; 168:474-85. https://doi.org/10.1111/ bjd.12149.

21. Herane MI, Ando I. Acne in infancy and acne genetics. Dermatology. 2003; 206:24-28. https://doi. org/10.1159/000067819.

22. Fernando MM, Stevens CR, Sabeti PC, Walsh EC, McWhinnie AJ, Shah A, Green T, Rioux JD, Vyse TJ. Identification of two independent risk factors for lupus within the MHC in United Kingdom families. PLoS Genet. 2007; 3:e192. https://doi.org/10.1371/journal.pgen.0030192.

23. Calemma R, Ottaiano A, Trotta AM, Nasti G, Romano C, Napolitano M, Galati D, Borrelli P, Zanotta S, Cassata A, Castello G, Iaffaioli VR, Scala S. Fc gamma receptor IIIa polymorphisms in advanced colorectal cancer patients correlated with response to anti-EGFR antibodies and clinical outcome. J Transl Med. 2012; 10:232. https://doi. org/10.1186/1479-5876-10-232.
24. Mellor JD, Brown MP, Irving HR, Zalcberg JR, Dobrovic A. A critical review of the role of Fc gamma receptor polymorphisms in the response to monoclonal antibodies in cancer. J Hematol Oncol. 2013; 6:1. https://doi. org/10.1186/1756-8722-6-1.

25. Purcell S, Neale B, Todd-Brown K, Thomas L, Ferreira MA, Bender D, Maller J, Sklar P, de Bakker PI, Daly MJ, Sham PC. PLINK: a tool set for whole-genome association and population-based linkage analyses. Am J Hum Genet. 2007; 81:559-75. https://doi.org/10.1086/519795.

26. Johansson A, Marroni F, Hayward C, Franklin CS, Kirichenko AV, Jonasson I, Hicks AA, Vitart V, Isaacs A, Axenovich T, Campbell S, Dunlop MG, Floyd J, et al, and EUROSPAN Consortium. Common variants in the JAZF1 gene associated with height identified by linkage and genome-wide association analysis. Hum Mol Genet. 2009; 18:373-80. https://doi.org/10.1093/hmg/ddn350.

27. Sakurai H. Targeting of TAK1 in inflammatory disorders and cancer. Trends Pharmacol Sci. 2012; 33:522-30. https:// doi.org/10.1016/j.tips.2012.06.007.

28. Mancari R, Portuesi R, Colombo N. Adult granulosa cell tumours of the ovary. Curr Opin Oncol. 2014; 26:536-41. https://doi.org/10.1097/CCO.0000000000000106.

29. Eichenfield LF, Del Rosso JQ, Mancini AJ, Cook-Bolden F, Stein Gold L, Desai S, Weiss J, Pariser D, Zeichner J, Bhatia N, Kircik L. Evolving perspectives on the etiology and pathogenesis of acne vulgaris. J Drugs Dermatol. 2015; 14:263-72.

30. Gottschalk TA, Tsantikos E, Hibbs ML. Pathogenic Inflammation and Its Therapeutic Targeting in Systemic Lupus Erythematosus. Front Immunol. 2015; 6:550. https:// doi.org/10.3389/fimmu.2015.00550.

31. Nimmerjahn F, Ravetch JV. Fcgamma receptors as regulators of immune responses. Nat Rev Immunol. 2008; 8:34-47. https://doi.org/10.1038/nri2206.

32. Van Cutsem E, Cervantes A, Adam R, Sobrero A, Van Krieken JH, Aderka D, Aranda Aguilar E, Bardelli A, Benson A, Bodoky G, Ciardiello F, D'Hoore A, Diaz-Rubio E, et al. ESMO consensus guidelines for the management of patients with metastatic colorectal cancer. Ann Oncol. 2016; 27:1386-422. https://doi.org/10.1093/annonc/mdw235.

33. Barrett JC, Fry B, Maller J, Daly MJ. Haploview: analysis and visualization of LD and haplotype maps. Bioinformatics. 2005; 21:263-65. https://doi.org/10.1093/ bioinformatics/bth457.

34. Gabriel SB, Schaffner SF, Nguyen H, Moore JM, Roy J, Blumenstiel B, Higgins J, DeFelice M, Lochner A, Faggart M, Liu-Cordero SN, Rotimi C, Adeyemo A, et al. The structure of haplotype blocks in the human genome. Science. 2002; 296:2225-29. https://doi.org/10.1126/science.1069424. 